\title{
LA TRADUCTION DE L'ALLUSION INTERTEXTUELLE
}

\author{
Katrien Lievois \\ katrien.lievois@uantwerpen.be \\ Université d'Anvers
}

\section{Résumé}

A partir d'un corpus constitué de La Chute d'Albert Camus, du Chagrin des Belges de Hugo Claus et d'Une année chez les Français de Fouad Laroui, ainsi que leurs traductions néerlandaises, cette contribution s'intéresse à la traduction de l'allusion intertextuelle ironique. Elle présente d'abord les concepts théoriques qui sous-tendent l'analyse, pour ensuite étudier plus en détail les 9 stratégies rencontrées : la traduction standard, la traduction littérale, la traduction avec marquage, la non-traduction, la traduction dans une troisième langue, les gloses, l'omission, la substitution par une intertextualité appartenant à la culture cible et la substitution par l'architextualité.

\begin{abstract}
"The Translation of Intertextual Allusion"

Based on a corpus including La Chute by Albert Camus, Le Chagrin des Belges by Hugo Claus and Une année chez les Français by Fouad Laroui, and their dutch translations, this contribution examines the translation or ironic intertextual allusions. First, it exposes the theoretical concepts on which the analysis is built; it follows up with a more detailed study of the nine encountered strategies: standard translation, literal translation, marked translation, non-translation, translation in a third language; explication, omission, replacement by intertextuality from the target culture and replacement by architextuality.
\end{abstract}

Mots-clés : Allusion. Intertextualité. Traduction littéraire. Ironie. Parodie.

Keywords: Allusion. Intertextuality. Literary translation. Irony. Parody. 
Manuscript received on June 30, 2016 and accepted for publication on November 16, 2016.

Para enlazar con este artículo / To link to this article:

http://dx.doi.org/10.6035/MonTI.2017.9.5

Para citar este artículo / To cite this article:

LiEVOIS, Katrien. (2017) "La traduction de l'allusion intertextuelle." In: Martínez Sierra, Juan José \& Patrick Zabalbeascoa Terran (eds.) 2017. The Translation of Humour / La traducción del humor. MonTI 9, pp. 125-148. 


\section{Introduction}

Même si la naissance de la notion d'intertextualité est souvent située dans les années 1970 et dans le groupe Tel Quel, la pratique de l'emprunt littéraire semble très ancienne et universelle, du moins dans la tradition occidentale, et a suscité de très nombreuses analyses de la part des spécialistes. Ce procédé littéraire, auquel on a donné des noms différents selon sa forme et sa fonction, est parfois même pointé comme une des caractéristiques mêmes de la littérarité (e.a. Compagnon 1979: 67; Riffaterre 1979). Quand on sait l'importance de la traduction pour la circulation du texte littéraire, l'on comprend aisément l'intérêt que peut avoir dans la traductologie littéraire la problématique de l'emprunt littéraire.

C'est pourquoi nous nous proposons, dans cette contribution, de nous pencher sur une forme spécifique de l'emprunt littéraire : l'allusion intertextuelle ironique. Il s'agira avant tout de préciser un certain nombre de concepts théoriques qui sous-tendent notre analyse (2). Ensuite, nous présenterons plus en détail les romans qui constituent le corpus de cette recherche pour ensuite (3) proposer les stratégies de traduction utilisées dans le cadre de la problématique qui nous intéresse.

\section{Concepts théoriques}

S'il nous est impossible dans cet article de retracer la naissance et l'évolution de la notion d'intertextualité, il convient cependant de pointer quelques moments importants dans cette histoire. C'est en réponse à la conception très large de l'intertextualité dans les travaux de Julia Kristeva (1969: 85) que Gérard Genette proposera une définition restreinte, qui fait, depuis lors, largement consensus. Genette préfère distinguer différentes formes de transtextualité, parmi lesquelles l'intertextualité, dont il précise qu'il s'agit d'« une relation de co-présence entre deux ou plusieurs textes [...], le plus souvent par la présence effective d'un texte dans un autre ». (1982: 8) Définis ainsi, le repérage et l'étude de l'intertextualité peuvent se faire à partir de passages relativement courts dans lesquels un emprunt littéraire est identifiable en tant que trace intertextuelle. 
Linscription de ce texte cité dans le texte citant peut, selon le critique, se réaliser des façons suivantes :

Sous sa forme la plus explicite et la plus littérale, c'est la pratique traditionnelle de la citation (avec guillemets, avec ou sans référence précise); sous une forme moins explicite et moins canonique, celle du plagiat (chez Lautréamont, par exemple), qui est un emprunt non déclaré, mais encore littéral ; sous forme encore moins explicite et moins littérale, celle de l'allusion, c'est-à-dire un énoncé dont la pleine intelligence suppose la perception d'un rapport entre lui et un autre auquel renvoie nécessairement telle pour telle de ses inflexions, autrement non recevable. (1982: 8)

Annick Bouillaguet (1989) développera explicitement les deux oppositions de Genette et ajoute à la citation (qui est littérale et explicite), le plagiat (littéral et non déclaré) et l'allusion (transformée et non-explicite), la quatrième catégorie qu'elle appelle la référence et qui est transformée et explicite. C'est à partir de ces quatre formes d'intertextualité que Geneviève Roux-Faucard proposera ses réflexions sur l'intertextualité et la traduction (2006).

Ces distinctions sont conceptuellement utiles et permettent en effet de fonder une recherche traductologique, car « face à la traduction, ces quatre types de traces ont des comportements relativement différents » (Roux-Faucard 2006: 104). Il nous semble toutefois important de les articuler avec d'autres notions qui ont trait à l'emprunt littéraire en vue d'une analyse des traductions.

Il peut également être utile de rappeler que ces différentes formes de traces intertextuelles (dont l'allusion n'est qu'une seule selon la terminologie de Genette) sont, dans leur ensemble, appelées allusions littéraires par des chercheurs qui se situent surtout dans un contexte anglo-saxon (e.a. Ben-Porat 1976; Coombs 1984; Perri 1978; Pucci 1998). C'est cette acception de la notion d'allusion que nous utiliserons dans notre analyse : nous nous intéresserons donc à toutes les traces intertextuelles, qu'elles soient non déclarées ou explicites, littérales ou transformées. Ces allusions sont cependant intertextuelles selon l'acception de Genette, c'est-à-dire qu'elles concernent la « présence effective d'un texte dans un autre » (1982: 8) et sont donc assez clairement identifiables. Cela nous permet en effet d'isoler les passages dans lesquels se déploient des allusions qui peuvent, à leur tour, fournir la base pour les analyses des traductions. Nous ne traiterons donc pas d'autres formes de transtextualité distinguées par Genette, comme l'hypertextualité, la métatextualité, l'architextualité ou la paratextualité.

Si le terme allusion littéraire est en effet largement employé, Ziva Ben-Porat insiste sur le caractère trompeur qu'il peut avoir (1976: 105-106). Il va de soi que ce type d'allusion n'apparaît pas seulement dans des textes littéraires d'une part et ne concerne pas uniquement des textes cités appartenant aux Belles 
Lettres de l'autre. De très nombreuses formes artistiques (e.a. la peinture, le cinéma, la photographie...) et textuelles (articles journalistiques, publicités, discours politiques... ) contiennent en effet des clins d'œil à d'autres textes et des œuvres littéraires également réfèrent souvent à des réalisations artistiques et textuelles non littéraires, comme des chansons, des films, des publicités, des phrases devenues cultes... Ritva Leppihalme, qui étudie la traduction de ce qu'elle nomme " allusive wordplay ", propose dans ce cadre aussi le terme " frame », qu'elle définit comme « a combination of words that is more or less fixed conventionnaly in the minds of a group of language users $[\ldots$,$] an$ example of preformed linguistic material » (1996: 200).

Ben-Porat (1976: 110-113) précise également qu'il existe différentes étapes dans l'identification et la reconnaissance d'une allusion littéraire. Il faut avant tout que le signe dans le texte citant soit reconnu comme une expression appartenant aussi à un texte cité. Le fait de reconnaître un signe comme allusion, implique souvent, mais pas toujours, que ce texte cité soit effectivement identifié. Létablissement de la relation entre ces deux textes, citant et cité, permettra peut-être, dans un troisième temps, de modifier la première interprétation locale ( Initial Local Interpretation») du signe allusif et, le cas échéant dans un quatrième moment, d'arriver à une interprétation renouvelée du texte citant dans son entièreté sur la base de ses relations avec le texte cité. Toutes ces étapes d'interprétation exigent une grande familiarité avec la tradition artistique et littéraire du texte dans lequel se déploie l'allusion. Dans le cadre de la traduction de l'allusion littéraire, l'identification et la reconnaissance peuvent en être fondamentalement modifiées dans la mesure où le texte source a été décontextualisé et que le lecteur du texte cible possède un autre univers de référence culturel. La décontextualisation est faible lorsqu'il s'agit de deux cultures proches, moyenne s'il existe déjà une traduction connue dans la culture cible du texte cité dans le texte source et à son maximum si le texte cité n'est pas connu dans la culture cible (Roux-Foucard : 2006, 106). Une analyse de la traduction de l'allusion s'intéressera donc à la façon dont se fait la recontextualisation de l'emprunt littéraire dans la culture cible (Roux-Foucard : 2006, 108).

Si l'intertextualité n'est pas nécessairement ironique, l'on sait combien l'ironie littéraire doit souvent à l'intertextualité en général et à l'allusion en particulier. Les emprunts littéraires dont nous étudierons les traductions sont tous à situer dans des œuvres essentiellement ironiques et dans lesquelles l'intertextualité contribue de manière importante à développer l'ethos ironique. Prises individuellement, les allusions ne sont donc pas à coup sûr humoristiques, ni ironiques. C'est davantage leur fonctionnement intertextuel comme procédé global qui les rend ironiques. 
Lironie peut en effet se déployer à l'aide de figures de style tout à fait explicites et appuyées comme l'oxymore, l'hyperbole, l'exagération, la surenchère, le mélange des styles, l'antiphrase... Lintertextualité est souvent considérée comme une façon plus subtile de créer l'ironie et, partant, comme un procédé plus délicat et raffiné dans ce cadre. Certainement pour ce qui est de l'ironie littéraire, l'avis est largement partagé que «l'ironie la plus discrète est toujours la meilleure, en d'autres termes, l'ironie est d'autant plus réussie qu'elle recourt à moins de signaux pour se faire reconnaître » (Schoentjes 2001: 158) C'est un élément auquel le traducteur aura à faire face non seulement en tant que lecteur du texte source, mais également en tant que producteur du texte cible. Poussé par la volonté de rendre sans équivoque aucune l'aspect ironique ou humoristique de l'intertextualité, il risque effectivement d'en effacer la subtilité :

[T] he translator may feel the need to turn covert forms of humor into more overt manifestations, especially if the translation is less effective than the original, in this case the translator conveys that there has been attempt at being funny, while acknowledging failure to render the actual funniness [...]. In any case, this kind of practice is quite common in translation on the whole, so much so that it has given rise to the hypothesis that translations have a universal tendency to be more explicit than their source texts. The down side of this practice occurs when humor is based, or relies on subtlety, tongue-incheek, irony, allusion and other such covert devices, but the translator resorts to broad brush, bluntness and denotative meaning to spell everything out to the text user in no uncertain terms, thus shredding the very fabric of this kind of humor. (Zabalbeascoa 2005: 190)

Nous étudierons donc la traduction d'allusions intertextuelles ironiques, telles qu'elles apparaissent dans des passages clairement identifiables. Pour ce faire, nous prendrons en compte la façon dont se réalise la recontextualisation de l'allusion du texte citant au texte cité dans le nouveau texte source.

\section{Présentation du corpus}

Comme points de départ de cette analyse, nous avons choisi 3 romans: $\mathrm{La}$ Chute d'Albert Camus (1956), Het verdriet van België (Le chagrin des Belges) de Hugo Claus (1983) et Une année chez les Français de Fouad Laroui (2010b).

La Chute est la dernière ouvre terminée par Camus et publiée de son vivant. Ce texte est sorti un an avant que l'auteur reçoive le prix Nobel de la littérature et Camus est à ce moment-là au sommet de sa célébrité. Il existe de ce texte à ce jour deux traductions en néerlandais : la première a été faite par Anne Maclaine Pont et publiée en 1957, une année à peine après la publication de l'original, et la seconde (1985) est de Dolf Verspoor. Pourquoi La Chute pourrait-il offrir un point de départ intéressant pour creuser la question qui 
nous intéresse ? Rappelons brièvement le sujet de cette œuvre : un Parisien rencontre un autre Parisien et engage la conversation avec lui. La thématique fondamentale de La Chute est celle de la culpabilité et de la perte de l'innocence et le discours que nous tient le narrateur, Jean-Baptiste Clamence, prend la forme d'une accusation de plus en plus sévère au fil des pages. Néanmoins, l'autocritique apparente de Clamence se transforme peu à peu en une critique et condamnation de l'homme moderne en général et de l'intellectuel français en particulier. Un des ressorts les plus importants du texte concerne précisément son ancrage prononcé dans la langue et la culture françaises : il présente de très nombreuses allusions culturelles et littéraires françaises. La Chute est également un texte fort apprécié d'un des auteurs français les plus canonisés.

Le deuxième roman que nous avons sélectionné jouit d'un prestige assez similaire dans le champ littéraire où il a vu le jour. Le chagrin des Belges de Hugo Claus est en effet l'œuvre majeure d'un auteur néerlandophone incontournable. Loriginal a été publié en 1983 et en 1985 Alain Van Crugten, un traducteur fort respecté dans le domaine linguistique néerlandais, l'a traduit en français. Het verdriet van België - littéralement le chagrin de la Belgique et non pas des Belges - est un roman d'apprentissage de 714 pages, qui est pour une bonne part autobiographique. Il raconte les années 1938 à 1947, qui sont celles du développement émotionnel et intellectuel du protagoniste, Louis Seynaeve. Lintertextualité du Chagrin des Belges joue un rôle important et a une valeur thématique significative. Le père de Louis est ce qu'on appelle en Belgique un «flamingant » : il œuvre pour l'émancipation du peuple flamand dans le contexte belge entre les deux guerres. La famille de Louis tend aussi à sympathiser avec les Allemands. Ainsi, en 1940, Louis, influencé par les idées de sa famille, laisse éclater sa joie quand « enfin » les Allemands envahissent la Belgique. Lintertextualité du Chagrin des Belges tend donc à montrer la supériorité de la culture et littérature allemandes et de ce que cette famille catholique et flamingante considérait comme la bonne littérature flamande, c'est-à-dire pour la plus grande part des auteurs d'extrême droite ou collaborateurs. En même temps, cet entourage germanophile et hypocritement catholique tend à donner une image dépréciative de la littérature française qui est considérée comme amorale et décadente. Cette thématique est déjà en soi, selon le principe du double regard, tout à fait ironique. Car si le narrateur peut avoir ces opinions nationalistes, ces idées ne sont évidemment (plus) celles de l'auteur, Hugo Claus, en 1983. Il nous semble donc que Le chagrin des Belges est, davantage qu'un roman d'apprentissage, un roman sur les mécanismes de l'endoctrinement nationaliste et que son intertextualité doit être évaluée dans ce contexte. 
Fouad Laroui est Marocain de naissance. Il a fait des études d'économie et il a suivi une formation d'ingénieur en France. Aujourd'hui il est professeur de littérature française et de culture arabe à l'université d'Amsterdam aux PaysBas, où il vit. Son œuvre est constituée d'une dizaine de romans et six recueils de nouvelles en français, dont la plupart ont été publiés aux Éditions Julliard. En 1996, au moment où il a commencé à publier ses romans en français, il séjournait depuis plusieurs années déjà aux Pays-Bas et avait obtenu la nationalité néerlandaise. Outre ses textes en français, il a publié également directement en néerlandais : un essai (2001b), des poèmes (2002b) et des nouvelles (2006). Son œuvre fictionnelle francophone quasi intégrale (1999, 2001a, 2002a, 2004, 2005, 2008, 2010a), dont le roman que nous soumettrons à l'analyse (2012), est également traduite en néerlandais. Son traducteur néerlandais jouit dans son domaine linguistique d'une très grande réputation. Frans Van Woerden doit cette considération essentiellement à ses traductions de Louis-Ferdinand Céline, qui lui ont valu en 1988 le prix Martinus Nijhoff, décerné chaque année à la meilleure traduction à partir du néerlandais ou vers cette langue. Une année chez les Français se déroule en 1969 et raconte la première année de Medhi Khatib dans le Lycée Lyautey à Casablanca. Si Medhi, un jeune garçon marocain de 10 ans d'origine campagnarde et modeste, est accepté au prestigieux Lycée français de Casablanca, un endroit destiné aux enfants de hauts fonctionnaires français, c'est grâce à une bourse du gouvernement français obtenue par son instituteur. Le roman décrit avant tout le choc culturel que Medhi doit affronter, le contraste entre sa vie au sein de sa famille de culture musulmane et celle au lycée où la culture française est évidemment largement présente et valorisée. Lintertextualité dans Une année chez les Français est donc constitutive de la thématique du roman. Cette œuvre n'a évidemment pas le même statut de canonisation que les autres textes que nous avons choisis, mais a cependant été retenue dans la première sélection du prix Goncourt de 2010. C'est plutôt le fait que Fouad Laroui est souvent classé dans la littérature transnationale, de l'immigration ou de la diaspora qui nous a poussée à l'inclure dans cette analyse. Il nous semble que ce type de textes peut présenter des cas d'intertextualité intéressants pour notre problématique.

Dans l'établissement de ce corpus, il était important de reprendre des romans dans lesquels l'intertextualité est intrinsèquement liée à la thématique. Elle y a donc un rôle et une fonction spécifiques qu'il conviendra de garder à l'esprit au moment d'en analyser les traductions. Nous avons également voulu que soient représentées les deux directions pour ce qui est de la traduction : aussi bien du français vers le néerlandais que l'inverse. 


\section{Comment traduire l'allusion intertextuelle ironique?}

\subsection{La traduction standard}

Lallusion intertextuelle est donc une suite de mots dans le texte citant qui réfère de manière directe ou implicite à un texte cité. Pour traduire cette allusion, il est logique de voir si le texte cité est traduit dans la langue cible et de reprendre cette traduction ou de s'en inspirer. Il s'agit là de la stratégie qui est souvent appelée standard (Leppihalme 1997: 83). Nous la rencontrons maintes fois quand il s'agit de traduire des allusions à des titres et à des passages d'œuvres littéraires importantes. Les exemples qui suivront tendent cependant à montrer qu'il n'est pas toujours facile de déterminer ni quelle serait la traduction standard d'une part, ni où se trouve la frontière entre la traduction standard et la traduction littérale de l'autre.

Il va en effet sans dire que certains textes ont été traduits de très nombreuses fois. L'exemple le plus connu dans ce cadre est incontestablement la Bible. Pour ce qui est des textes français traduits à plusieurs reprises, l'on pourrait également citer Le Cid de Corneille ou Les Fables de Jean de La Fontaine, deux cas d'intertextualité que nous rencontrons dans le roman de Fouad Laroui. L'auteur fait en effet de très nombreuses allusions à de nombreux poètes célèbres - citons encore François Villon, Joachim du Bellay, Alphonse de Lamartine, Victor Hugo, Charles Leconte de Lisle, Arthur Rimbaud, Paul Valéry, ... - mais l'auteur qui constitue sa source privilégiée est sans conteste Jean de La Fontaine. Les allusions au célèbre fabuliste ne sont pas seulement nombreuses - il y en a six -, mais il s'agit aussi chaque fois d'extraits relativement longs. Toutes ces allusions à la poésie française ont des marqueurs pour en faciliter la reconnaissance, le plus souvent des guillemets. Le traducteur a repris les mêmes marqueurs ; il signale donc de la même façon la présence d'allusions à la poésie française. Pour sa traduction de l'allusion au Cid - « La valeur n'attend pas le nombre des centimètres » (Laroui 2010b: 196) qui est un clin d'œil au vers de Corneille : « Aux âmes bien nées, la valeur n'attend point le nombre des années $»-$, mais sans doute surtout pour celle d'un long extrait de la tragédie que les écoliers mettent en scène lors de leur cours de théâtre, Van Woerden indique au début du volume qu'il s'est basé sur la traduction de Laurens Spoor. Il en fait de même pour les allusions à La Fontaine : il précise en effet qu'il a employé les traductions de Jan van den Berg.

Il est parfois impossible de faire la distinction entre une traduction standard et une traduction littérale. Dans le Chagrin des Belges, le personnage principal rêve de devenir écrivain et se compare à des auteurs belges connus. Ainsi : 
(Ex.1) Kaas van Elsschot is korter. (1983: 714)

Fromage d'Elsschot est encore plus court. (1985: 827)

La nouvelle Fromage est un des textes classiques dans le domaine néerlandais, mais la traduction française, faite par Xavier Hanotte, n'a vu le jour qu'en 2003. Même si à strictement parler, Van Crughten a donc traduit littéralement cette allusion, parce que Fromage n'avait pas encore vu le jour quand il travaillait au Chagrin des Belges, il s'avère donc peu utile de faire ici une distinction entre la stratégie de la traduction standard et celle de la traduction littérale.

Dans La Chute, Clamence compare Amsterdam à l'enfer et fait ainsi une allusion au chef-d'œuvre de Dante. Pour rendre cette intertextualité, le traducteur utilise également l'expression employée dans les traductions néerlandaises de La Divine Comédie : « hellekringen ».

(Ex.2) Avez-vous remarqué que les canaux concentriques d'Amsterdam ressemblent aux cercles de l'enfer? (1962: 1483)

Is het $u$ opgevallen dat de grachtengordels van Amsterdam overeenkomen met de hellekringen? (1985: 610)

La première traductrice de La Chute n'avait pas utilisé cette expression, mais avait proposé une traduction littérale de l'expression française :

Is het $\mathrm{u}$ wel eens opgevallen, dat die concentrische grachten van Amsterdam op de cirkels [cercles] van de hel [de l'enfer] lijken? (Camus 1957: 16)

Si nous partons de l'idée que l'allusion est en effet un " a signifiant, whose signifié [is] reveiled in the merging of two texts by one sign " (Pucci 1998: 16), l'intertextualité ne se présente sans doute pas de la même façon dans le texte cible que dans le texte source, dans la mesure où le lecteur néerlandais ne reconnaîtra vraisemblablement pas une allusion à Dante à partir de la traduction littérale de « cercles de l'enfer ».

La suite de ce passage nous montre encore comment une traduction littérale peut en effet entièrement effacer l'allusion intertextuelle :

Ici, nous sommes dans le dernier cercle. Le cercle de... Ah ! Vous savez cela ? Diable, vous devenez plus difficile à classer. (Camus 1962: 1483)

Ce que Clamence ne dit pas, mais que son interlocuteur semble savoir, c'est que le dernier cercle de l'Enfer de Dante est celui des traîtres et plus spécifiquement celui où demeure Lucifer (auquel de toute évidence le titre La Chute fait aussi explicitement référence). Lauto-ironie de Clamence est subtile : l'intertextualité 
montre combien il s'estime un intellectuel cultivé, mais le fait de se placer dans le même cercle que Lucifer indique qu'il se considère par la même occasion comme un traître et souligne qu'il est conscient de sa culpabilité.

Voici les deux traductions néerlandaises de ce passage :

Hier zitten we in de allerbinnenste cirkel. De cirkel der... Aha, weet u dat al? Deksels, ik kan u hoe langer hoe moeilijker plaatsen. (Camus 1957: 16) $\rightarrow$ [bon sang]

Hier zitten we in de binnenste kring. De kring van de ... Hé, dat weet u? Verdraaid, ik kan u steeds moeilijker thuisbrengen. (Camus 1985: 610) $\rightarrow$ [nom d'un chien]

Si Clamence exprime son étonnement à l'aide de l'expression diable, ce n'est évidemment pas un hasard. Il existe en néerlandais une expression d'étonnement dans laquelle apparaît le diable ( « verduiveld »), mais aucun des traducteurs ne l'a utilisée. Ils ont préféré une expression qui rend plutôt « bon sang » et " nom d'un chien ». Il s'agit là sans doute d'un exemple qui montre que la difficulté de la traduction réside autant et peut-être davantage dans la reconnaissance et l'identification de l'allusion intertextuelle dans le texte source que dans la production de l'ironie dans le texte cible.

Dans Une année chez les Français, Régnier, un des surveillants du Lycée de Lyautey, qui préfère pour indiquer sa fonction le terme de "pion », car il est conscient qu'il fait partie de ceux qui sont «des pions dans le système de bourrage de crâne, des pions dans le Système » (Laroui 2010b: 109) cite l'Internationale. L'ironie du passage réside dans le fait que Régnier essaie de montrer à Mehdi qu'ils appartiennent tous deux à la même classe sociale, les prolétaires et les opprimés, mais utilise pour exprimer leur solidarité des notions et des expressions que le petit Marocain ne comprend absolument pas : " C'est quoi, un pro-lait-terre ? (Laroui 2010b: 110)». Mehdi interprète ces termes, "prolétaires » et " damnés de la terre », comme autant d'insultes de la part du surveillant. Pour rendre cette allusion, le traducteur reprend la version néerlandaise la plus connue de ce chant révolutionnaire, qui a d'ailleurs été faite par une des grandes poétesses néerlandaises, Henriette Roland Holst :

(Ex. 3) ... nous sommes les damnés de la Terre! (Laroui 2010b: 110) ... we zijn de verworpenen der aarde! (Laroui 2012: 97)

Comme c'est le cas dans de nombreuses autres langues, il existe plusieurs traductions néerlandaises de l'Internationale. Il est logique que le traducteur choisisse la plus couramment employée et donc celle que le lecteur du texte cible aura le plus de chances de reconnaître. 


\subsection{La traduction littérale}

La stratégie de la traduction standard est souvent applicable quand les allusions concernent des œuvres littéraires, qui sont en effet régulièrement traduites. Nous constatons cependant des différences importantes d'après la langue de l'original. Depuis les travaux de Lawrence Venuti $(1995,1998)$ d'abord et des sociologues de la traduction (Heilbron 1999, 2000, 2008; Heilbron \& Sapiro 2007) ensuite, nous savons qu'il existe de grandes divergences numériques entre les traductions à partir des différentes langues (extraduction) et vers ces langues (intraduction). Pour le couple qui nous intéresse, le français et le néerlandais, l'écart est grand. Ainsi, quand on consulte l'Index Translationum (recherche effectuée le 23/06/2016) pour voir combien de textes littéraires ont été traduits entre 1950 et 2015 du français en néerlandais, le résultat est 6823 ; si l'on fait la même recherche, mais en inversant la direction, on obtient 1466. Il nous semble que c'est là le principe qui explique pourquoi l'on trouve tellement plus d'exemples de traductions standard dans les traductions d'originaux français que dans Le chagrin des Belges. Pour reprendre les distinctions établies par Roux-Foucard (2006: 108), on voit que dans le cas des traductions de textes français en néerlandais, la décontextualisation est souvent faible ou moyenne; les traductions françaises de textes néerlandais relèvent davantage d'une décontextualisation maximale.

La traduction littérale s'impose également quand l'allusion concerne un genre textuel qui n'est que rarement traduit, ainsi entre autres les discours politiques ou les chansons. Une des phrases-clés les plus connues des discours politiques de Charles de Gaule est « Je vous ai compris ! », prononcée en 1954, en pleine Guerre d'Algérie. Cette formule, qui est restée dans les annales de la politique française, est parfois connue à l'étranger sous sa forme originelle, mais ne possède évidemment pas de traduction standard. Chez Laroui (2010b : 73), le surveillant général l'utilise pour réagir à une situation tout à fait terre à terre, le traducteur la traduit donc littéralement ( "Ik heb het begrepen! », Laroui 2012 : 66) et efface ainsi l'allusion intertextuelle.

\subsection{La traduction avez marquage}

Les chansons également franchissent les frontières plus souvent dans la langue originale qu'en traduction. L'exemple de l'Internationale (Ex.3) constitue en effet une exception, parce que dans la plupart des cas, les chansons ne sont pas traduites. Il s'ensuit que Van Woerden n'a pu se baser sur une traduction standard pour rendre les allusions à des chansons françaises dans Une année chez les Français : 
(Ex. 4) ... et tout cela fait d'excellents Français! (Laroui 2010b: 67)

... en dat waren allemaal eersteklas Fransen! (Laroui 2012: 61)

(Ex. 5) Les aristocrates, à la lanterne ! (Laroui 2010b: 88)

Aan de lantaren met die aristocraten! (Laroui 2012: 79)

(Ex. 6) Tiens bon la vague et tiens bon le vent... Hissez haut! Santi-a-a-no

Si Dieu veut, toujours droit devant, nous irons jusqu'à San Francisco

! (Laroui 2010b: 219)

Hou zee en hou goeie wind... Hijsen-hi, hijsen-ho! Santi-ááá-no!

Met Gods ge-nááá Tot in... San Francis-cooo! (Laroui 2012: 192)

D'y penser j'avais le cœur gros

En doublant les feux de Saint-Milo (Laroui 2010b: 223)

Daaraan denk ik keer op keer Met een hart zo vol met zeer, Oho! oho!

bij het zien van de vuurtoren van Saint-Malo...oho! (Laroui 2012: 97)

Dans ces trois cas, il s'agit de chants et de chansons très connus dans la culture source. Ça fait d'excellents Français est une chanson interprétée par Maurice Chevalier en 1939 visant à remonter le moral des Français qui venaient d'être mobilisés. Si un des surveillants du lycée français y fait allusion devant le monument aux morts de l'école, ce n'est pas tant pour expliquer qu'en temps de guerre, les Français oublient leurs vies douillettes et leur divergences politiques, mais plutôt pour montrer que les anciens professeurs et élèves qui s'étaient battus pendant la Deuxième Guerre mondiale pour la France étaient d'origines très diverses : Italiens, Allemands, Juifs, Roumains... Ah ! ça ira, ça ira, ça ira, est un des premiers chants révolutionnaires et peut être considéré en quelque sorte comme le symbole même de la Révolution. Dans le cadre d'une simple querelle d'adolescents, Ramòn, un élève espagnol qui ne fait pas de secret de ses sympathies républicaines, l'entonne pour intimider un camarade arabe, issu d'une riche famille de propriétaires terriens. Santiano, enfin, est une des chansons les plus populaires de Hugues Auffrey. Elle est sortie en 1961, pour se retrouver immédiatement en tête de tous les hit-parades et depuis lors, sa popularité ne s'est jamais démentie. Elle apparaît d'ailleurs dans la plupart des paroliers de chants de camp et de veillée. Dans le roman de Laroui, la chanson est convoquée pour exprimer le chagrin du petit Marocain - « D'y penser j'avais le cœur gros »-quand il comprend que, malgré la gentillesse dont font preuve les parents de son meilleur ami (français) entre autres en l'invitant pour une sortie en bateau, jamais ils ne le considéreront vraiment comme un des leurs. 
Même si le traducteur trouve des équivalents heureux pour ces formules, il ne pourra évoquer auprès de son lecteur le même sentiment qui naît chez le lecteur francophone qui reconnaît et identifie ces allusions dans le texte source. Il y a en effet sans doute peu de genres textuels qui nous touchent aussi profondément que certaines chansons. Par le fait qu'elles appartiennent souvent à l'époque de notre enfance, les chansons doublent le plaisir intellectuel de la reconnaissance de l'allusion en y ajoutant une composante émotionnelle. Reste que la traduction peut partiellement rendre la structure formelle de l'allusion ou inclure un jeu de mots pour marquer l'expression employée. Dans l'exemple 4 Van Woerden établit implicitement un lien avec le monde de la guerre. "Eersteklas » (littéralement « de première classe ») équivaut évidemment à " excellents », mais constitue en même temps un clin d'œil $\mathrm{au}$ " soldat de première classe ", un grade militaire, et réfère ainsi au monde de l'armée. Pour rendre Santiano, le rythme de la chanson de marins qui est indiqué une seule fois en français (Santi-a-a-no), est répété dans le texte cible (Gods ge-nááá; San Francis-cooo!) et renforcé par des cris typiques du genre (Hijsen-hi, hijsen-ho ; Oho! oho!)

\subsection{La non-traduction}

Il va sans dire que le domaine linguistique néerlandophone, et plus particulièrement la Belgique néerlandophone, a une meilleure connaissance de la culture française que ce n'est le cas dans le sens inverse. Nous retrouvons d'ailleurs plusieurs allusions intertextuelles en français dans Het verdriet van België, qui restent telles quelles dans le texte cible. Ces allusions concernent aussi bien des référents culturels français (comme la chanson Malbrouck s'en va-t-en-guerre, p. 688 et le vers issu de Cinna de Corneille : "si l'on doit le nom d'homme à qui n'a rien d'humain, à ce tigre altéré de tout le sang romain ", p. 170), que plus largement internationaux, mais qui étaient, dans la Belgique du milieu du $20^{\text {ième }}$ siècle, plus connus en français (ainsi Toujours sourire, le cour douloureux, p. 143, la traduction de l'air Immer nur lächeln de l'opérette Das Land des Lächelns de Franz Léhar). On voit ainsi qu'elles appartiennent aussi bien à la culture haute qu'à celle plus populaire. Pour un passage, notamment une allusion à une chanson de Félix Mayol qui date de 1902, le traducteur indique en note de bas de page « en français »:

(Ex.7) In uw conversatie, mon cher, hoor ik het air dat ik zong in de loopgraven in Veertien-Achttien, 'Viens, poupoule, viens, poupoule, viens!' (1983: 430) 
Dans votre conversation, mon cher (note en bas de page : en français), j'entends l'air que je chantais dans les tranchées de quatorze-dix-huit : Viens Poupoule, viens Poupoule, viens! (note en bas de page: en français) (1985: 499-500)

Il convient de souligner que dans 3 des 4 cas de non-traduction que nous avons isolés, il s'agit de chansons. Le seul exemple de cette stratégie pour La Chute, qui est d'ailleurs employée dans les deux versions néerlandaises (Camus 1957: 37; 1985: 625), concerne également une chanson, datant de 1912, mais qui avait été rendue très populaire à la fin des années ' 40 par Tino Rossi : Femmes, que vous êtes jolies! (Camus 1962: 40)

Pour le roman de Laroui, les cas de non-traduction concernent cependant des références à la littérature française : Vipère au poing (2012: 64) réfère au roman de Hervé Bazin et Le théâtre et son double à Antonin Artaud. (2012: 65)

La non-traduction ou la copie verbatim (Desmet 2001: 34) de l'allusion est également employée pour les passages où entre en ligne de compte une troisième langue, qui est ni celle du texte source, ni celle du texte cible. Cette stratégie est envisageable quand cette troisième langue a un statut privilégié et est comprise aussi bien par le lecteur du texte source que du texte cible. Dans Une année chez les Français, les exemples concernent tous l'anglais ou le latin.

(Ex. 8) I shall return! (2010b: 27)

I shall return! (2012: 24)

(Ex. 9) Dulce et decorum est pro patria mori. (2010b: 66)

Dulce et decorum est pro patria mori. (2012: 59)

(Ex.10) De profundis, morpionibus... (2010b: 91)

De profundis, morpionibus... (2012: 82)

Que ce soit une phrase-clé du général américain Douglas MacArthur (Ex.8), les lignes d'une des Odes d'Horace (Ex.9) ou le début du refrain d'une chanson paillarde écrite en français et dont les paroles sont de Théophile Gautier (Ex. 10), les allusions restent telles quelles dans la traduction.

\subsection{La traduction dans une troisième langue}

Pour un passage, nous constatons qu'une troisième langue peut être utilisée pour rendre une allusion qui avait été formulée en français, la langue du texte source : 
(Ex.11)La Petite musique de nuit de Mozart (Laroui 2010b: 211) Eine kleine Nachtmusik van Mozart (Laroui 2012: 184)

La première fois qu'apparaît cette référence à Mozart elle n'est pas ironique, mais sert seulement à représenter la famille française d'un ami de lycée de Mehdi, qui fête le Réveillon de Noël et boit du champagne ou du chocolat chaud en écoutant de la musique classique, tranquillement assise ensemble au salon. Répétée à de très nombreuses reprises, celle allusion acquerra au fur et à mesure un caractère ironique, même s'il s'y mêle toujours en même temps la mélancolie et la nostalgie. Et si la Sérénade $n^{\circ} 13$ en sol majeur de Mozart est en effet mieux connue en français sous le titre Une petite musique de nuit, tel n'est pas le cas du néerlandais qui reprend plutôt le titre en allemand.

\subsection{Les gloses}

Quand la décontextualisation est grande, le traducteur devra veiller à une vraie recontextualisation dans la culture cible. Pour ce faire, il peut faire appel à différentes formes de marquage et d'explications. Lajout de majuscules, d'italiques et de guillemets peut en effet guider le lecteur pour ce qui est de la reconnaissance d'une allusion. En vue d'orienter l'identification de l'emprunt littéraire, l'on peut prévoir des notes de bas de page ou de fin de volume, des glossaires, des explications dans une préface ou une postface, ... Les gloses qui prévoient des indications concernant l'identification devront cependant être utilisées de façon réfléchie : d'une part, elles favorisent effectivement une réception plus subtile de l'œuvre, mais de l'autre, si elles sont trop systématiquement employées, elles risquent de gâcher une partie du plaisir du lecteur. Car, comme le rappelle Linda Hutcheon, comprendre l'ironie, ainsi que les procédés qui lui sont propres et les phénomènes qui se trouvent à ses frontières, participe également de la « intellectual satisfaction », « including the delight in one's own interpretative virtuosity » que nous procure la reconnaissance d'une allusion ironique, allant même jusqu'à « feeling a certain pleasure of superiority » (1994: 42). Si le lecteur a l'impression que l'ampleur de sa culture générale ou sa subtilité littéraire ne sont pas reconnues, il goûtera nettement moins la traduction qu'il est en train de lire.

Les gloses peuvent être insérées pour expliciter aussi bien l'allusion intertextuelle même, que son aspect humoristique.

Certains clins d'œil intertextuels sont en effet constitutifs de la thématique d'un roman et doivent, sous peine de quasi-illisibilité du texte, être compris par le lecteur. Un des éléments importants du Chagrin des Belges concerne 
le nationalisme flamand. Certaines références le concernant seront en effet explicitées dans une note de bas de page.

(Ex.12) [H]ij die altijd zei: 'Alles voor Vlaanderen, Vlaanderen voor Christus!' (1983: 607)

[L] ui qui criait toujours Alles voor Vlaanderen, Vlaanderen voor Kristus! (1985: 704)

Van Crughten ne traduit pas ce slogan, qui est parmi les plus célèbres du mouvement flamand. Dans la mesure où ce mouvement est prioritairement dirigé contre les francophones, une traduction serait effectivement presque un non-sens. Il complète cette non-traduction cependant par une note en bas de page disant : «'Tout pour la Flandre, la Flandre pour le Christ !', slogan des nationalistes flamands. » (1985: 704)

Dans d'autres cas, c'est plutôt le caractère humoristique de l'allusion qui sera explicité par la note de bas de page. Ainsi dans La Chute, quand Clamence reprend un jeu de mots attribué au Christ dans le Nouveau Testament :

(Ex.13) Pierre, vous savez, le froussard, Pierre, donc, le renie : "Je ne connais pas cet homme... Je ne sais pas ce que tu veux dire...etc." Vraiment, il exagérait ! Et lui fait un jeu de mots: "Sur cette pierre, je bâtirai mon église". On ne pouvait pas pousser plus loin l'ironie, vous ne trouvez pas? (Camus 1956: 122)

La traductrice ajoute une note de bas de page :

De woordspeling Pierre-Petrus en pierre-rots gaat in het Nederlands uiteraard verloren. (Camus 1957: 117-118)

[Le jeu de mots Pierre-l'apôtre et Pierre-roc se perd évidemment en néerlandais.]

En effet, dans la traduction néerlandaise de l'Évangile selon Mattieu, il n'y a aucun jeu de mots, contrairement à la version française, mais seulement l'emploi d'une métaphore :

Ik zeg jou: jij bent Petrus*; op die steenrots zal Ik mijn kerk bouwen. (De Bijbel: Willibrordvertaling 1995, Matt. 16,18)

[Eh bien, moi, je te le déclare : tu es Pierre, le rocher sur lequel Je construirai mon église]

Dans certaines traductions cependant, dont la Traduction Willibrord citée supra, le jeu de mots est également expliqué. 


\subsection{L'omission}

Il est évidemment possible d'omettre simplement l'allusion dans la traduction. Reste que mon corpus présente très peu d'exemples de cette stratégie. Dans Le chagrin des Belges, Louis rend compte de ses activités pendant un séjour chez sa famille:

(Ex.14)Vijftiende dag. Emma zegt dat ik vuil ben als een jood. [...] [quinzième jour]

Zestiende dag. [seizième jour] Ik heb de HJ 'Ouwe Taaie, jippiejippiejee' geleerd. Zij denken dat het een Vlaams volkslied is. [...] Achttiende dag. [dix-huitième jour] [....]

Negentiende dag. [dix-neuvième jour] Wat een Geschichte. (1983: 451)

Le seizième jour, il fait référence à une chanson qui est souvent chantée par des enfants chez les scouts ou en excursion scolaire, mais les membres de sa famille croient qu'il s'agit d'une sorte d'hymne national flamand. Lironie pointe dans deux directions à la fois : non seulement les ruraux chez qui il loge ne connaissent pas la musique en vogue, mais surtout, ils ramènent tout à la seule idée qui les intéresse, celle de de l'importance de la culture flamande. Aussi bien la référence à cette chanson appartenant à la culture populaire que la remarque ironique concernant l'interprétation de la famille demanderaient en effet des explications un peu longues pour le lectorat francophone. Elles tombent dans la traduction, au même titre que deux jours de vacances :

Quinzième jour : Emma dit que je suis sale comme un Juif. [...]

Seizième jour. Quelle histoire! (Claus 1985 : 524)

\subsection{La substitution par une intertextualité appartenant à la culture cible}

Lallusion intertextuelle concerne donc la présence effective d'un autre texte (cité) dans un texte citant. Pour rendre ce type de fonctionnement littéraire, il est envisageable de remplacer le texte cité du texte source par un autre texte cité appartenant à la culture cible. Bogaert (2001: 13-17) analyse cette stratégie dans le cadre des traductions d'Alice aux pays des merveilles de Lewis Carroll et Roux-Faucard (2006: 107) pour celles du Pendule de Foucault d'Umberto Eco.

Chez Laroui, on lit par exemple le terme « la chienlit» (2010b: 60), référant dans l'esprit des Français directement au Général de Gaulle, qui a utilisé cette expression pour juger négativement les événements de mai '68. Frans Van Woerden remplace ce clin d'œil par « de grote kladderadatsch » (Laroui 2012: 55), un terme plus couramment utilisé en allemand, et également par Marx et 
Engels, pour indiquer la chute du système qui mènera au désordre et au chaos. Quand Claus réfère à « rijen rijen op een wagentje" (1983: 546), une chanson enfantine néerlandaise très populaire, son traducteur la remplace par « Hue hue à dada » (1985: 633), qui a statut tout à fait similaire dans la culture française.

Si la stratégie de la substitution de l'intertextualité semble à première vue une solution élégante au problème qui nous intéresse, elle ne peut cependant être employée systématiquement.

Tout d'abord, un usage trop récurrent du remplacement de l'allusion risque d'avoir pour conséquence une véritable relocalisation du roman. Ainsi, nous avons vu que le traducteur de Fouad Laroui utilise des stratégies diverses pour transposer les allusions à des chansons françaises, mais pas de substitutions. Le narrateur vit au Lycée de Casablanca et baigne dans la culture française. Remplacer les chansons citées françaises par des équivalents néerlandais irait à l'encontre de l'ancrage géographique du roman.

Il convient également d'évaluer la finalité de l'intertextualité. Celle-ci peut en effet être admirative, neutre, ironique, sarcastique ou même hostile. Pour ce dernier cas, Hatim et Mason vont jusqu'à parler de contratextualité (1990: 130-131). Il est important de garder à l'esprit la valeur de l'allusion quand on cherche une intertextualité correspondant dans la culture cible. Un passage du roman de Claus nous en offre un exemple intéressant. À un moment donné, l'on demande au personnage principal de réciter une partie d'un poème de Guido Gezelle, qui est souvent considéré en Flandre comme le plus grand poète du $19^{\text {ième }}$ siècle. Gezelle, un prêtre catholique, était pour Hugo Claus cependant aussi le symbole même du Flamand conservateur et a raillé à plusieurs reprises la vénération qu'on lui voue dans son pays. Dans Le chagrin des Belges, Louis commence en effet par réciter le début de "Het Schrijverke », un des poèmes très connus du poète brugeois. Mais quand on lui demande de répéter, il en présente une parodie scatologique et personne ne remarque la différence.

(Ex.15)Ik heb vanavond: 'O krinklende winklende waterding, met 't zwarte kabotseken aan', enz. gereciteerd. Ik moest het nog eens doen en zei: 'O stinkende, winkelende paterding, schart er uw rokske maar aan.' (1983: 451)

Ce n'est qu'en 2011 que ce poème a été traduit sous le titre "Le Tourniquet » et voici les vers concernés en français :

Ô chose tournoyante et louvoyante, bête aquatique au noir chapeau. 
Le texte cité qui fonde l'emprunt littéraire est d'une part fort valorisée dans la culture source, mais tourné en ridicule par le narrateur, qui dit quelque chose comme " Ô petite chose puante et louvoyante du cureton, frottes-y ta jupe ». Lironie est aussi bien parodique que satirique, car elle se dirige non seulement contre Gezelle en tant que poète, mais aussi en tant que représentant du catholicisme flamand auquel on reprochait des travers comme le manque d'hygiène corporelle et l'hypocrisie sexuelle.

Dans sa recherche d'une intertextualité équivalente, le traducteur devrait prendre en compte l'ambiguité entre le statut littéraire dont jouit traditionnellement Gezelle et la façon dont le représente Claus. En plus, faire réciter au personnage principal du Chagrin des Belges un poème français, constituerait presque un contresens, dans la mesure où c'est précisément la littérature française, ainsi que tout ce qui est français, qui est rejetée par la famille flamingante de Louis Seynaeve.

\subsection{Le remplacement par l'architextualité}

Van Crugten explique lui-même que, pour traduire ce poème et sa parodie, il fallait en fait « inventer un extrait de poème ressemblant vaguement à une traduction de Gezelle » (1989: 30) Il s'éloigne en effet de Gezelle et introduit quelques vers convenus et banalement lyriques. Il développe ensuite une parodie sur ce poème forgé en y introduisant également des éléments scatologiques.

Ce soir, je leur ai récité : 'O murmurante chuchotante source douce avec tes reflets noirs moirés' etc. On m’a demandé de le répéter et j’ai dit : 'O purulente et chiante soupe aux choux avec tes reflets merdorés.' (1985: 524)

Dans ce cas, le traducteur a substitué un pastiche du genre à l'allusion intertextuelle et l'intertextualité est dès lors remplacée par l'architextualité (Genette 1982: 8). Cette solution se présente également quand l'allusion ne concerne pas une œuvre spécifique, mais un genre littéraire particulier à la culture source. Quand le petit Mehdi est accueilli dans son nouveau lycée dans Une année chez les Français, l'on se rend compte qu'il n'a pas apporté assez de vêtements. Le directeur et un des professeurs essaient de trouver une solution.

(Ex.16)- Il lui manque... il lui manque la moitié des affaires ! Des chaussettes, des mouchoirs...

- Peut-être un cul-de-jatte qui jamais s'enrhume?

M. Lombard réprima un sourire.

- Ah, ah, très drôle... Et bravo, c'est un alexandrin. (2010b: 22) 
Lironie de ce passage réside évidemment dans la rupture de style qui consiste à réagir à une situation aussi prosaique au moyen d'une formé poétique traditionnellement réservée aux sujets graves. Lallusion littéraire est présentée comme explicitement humoristique (sourire - drôle), or, l'alexandrin ne fait pas partie des versifications courantes dans la poésie néerlandaise. L'humour de l'emploi d'un alexandrin ne passera donc pas dans la traduction, où ce sont des rimes plates à caractère comique qui valent à celui qui les crée, «monsieur le poète », les félicitations de son collègue :

'Wat zal ik mokken zonder sokken, want ben ik van boven goed... dan kan ik ook wel zonder ondergoed ! (...) Bravo, meneer de dichter. (2012: 20) [Félicitations, monsieur le poète]

\section{Discussion des résultats et conclusion}

La lecture et l'analyse attentives des allusions intertextuelles ironiques en traduction semblent indiquer qu'il est difficile de tirer des conclusions quantitatives concernant les types d'allusions et les stratégies employées. Deux éléments apparaissent comme plus importants pour évaluer les stratégies de traduction : l'importance thématique de l'allusion dans le texte source et le degré de recontextualisation nécessaire du texte cible.

Ce n'est pas tant la forme de l'allusion qui tend à influencer la stratégie utilisée, mais plutôt la signification qu'elle revêt dans la construction du sens dans le texte source. Si elles appartiennent au cœur de l'œuvre littéraire pour ce qui est de son contenu, elles seront traduites de façon plus explicite. Il est bien sûr difficile de quantifier l'importante thématique de certaines formes d'intertextualité pour le texte analysé, mais il nous semble que l'on peut trouver des exemples comparables et ainsi proposer des analyses fondées. C'est pour cette raison que nous n'avons inclus dans notre corpus que des œuvres dans lesquelles l'intertextualité était cruciale dans le cadre de l'interprétation globale du texte. C'est aussi par rapport à ce critère que l'on pourra analyser les stratégies qui concernent le remplacement par une intertextualité appartenant à la culture cible et la substitution par l'architextualité.

Un texte appartenant à une culture source qui est relativement bien connue par la culture cible, et dont de nombreux textes sont effectivement traduits dans la langue cible, nécessite moins de stratégies explicites que dans le cas contraire. Quand le texte source appartient à une culture moins bien connue par la culture du texte cible, le traducteur devrait veiller à une recontextualisation plus grande et plus dirigée. Dans le premier cas, on trouvera plus de 
traductions standard et plus de cas de non-traduction. Dans le deuxième cas, il faudra davantage de gloses et l'on rencontrera également plus d'omissions.

Il va de soi que l'analyse de 3 romans ne permet de présenter des résultats définitifs. Elle peut cependant fonder des analyses ultérieures qui devraient cependant, il semblerait, prendre en compte les deux éléments dégagés : l'importance thématique de l'allusion dans le texte source et le degré de recontextualisation nécessaire du texte cible.

\section{Références bibliographiques}

BEN-PORAT, Ziva. (1976) "The poetics of literary allusion." PTL: A journal for descriptive poetics and theory of literature, 1, pp. 105-128.

BOGAERT, Maarten. (2001) "Vertalen in Wonderland: De vertaling van de parodie." Linguistica Antverpiensia 35, pp. 7-22.

Bouillaguet, Annick. (1989) "Une typologie de l'emprunt." Poétique 80, pp. 489-497.

Camus, Albert. (1956) La Chute. Paris: Gallimard.

CAmus, Albert. (1957) De Val. Amsterdam: Bezige Bij.

CAmus, Albert. (1962) La Chute. Paris: Gallimard.

CAmus, Albert. (1985) De Val. Amsterdam: Bezige Bij.

Claus, Hugo. (1983) Het verdriet van België. Amsterdam: De bezige bij.

Claus, Hugo. (1985) Le chagrin des Belges. Paris: Julliard.

COMPAGNON, Antoine. (1979) La seconde main ou le travail de la citation. Paris: Seuil.

Coombs, James H. (1984) "Allusion defined and explained." Poetics 13:6, pp. 475-488.

De BIJBeL. (1995) Willibrordvertaling. <http://www.bijbel.net/wb/?b=35>.

DESMET, Mieke K. T. (2001) "Intertextuality/Intervisuality in Translation: The Jolly Postman's Intercultural Journey from Britain to the Netherlands." Children's Literature in Education 32:1, pp. 31-43.

GenetTe, Gérard. (1982) Palimpsestes: la littérature au second degré. Paris: Seuil. HATIM, Basil \& Ian Mason. (1990) Discourse and the translator. Londres: Longman. HEILBRON, Johan. (1999) "Towards a Sociology of Translation. Book Translations as a Cultural World-System." European Journal of Social Theory, 2:4, pp. 429-444. HeIlbron, Johan. (2000) "Translation as a cultural world system." Perspectives: Studies in Translatology 8:1, pp. 9-26.

HEILBRON, Johan. (2008) "Lévolution des échanges culturels entre la France et les Pays-Bas face à l'hégémonie de l'anglais Translatio. Le Marché de la traduction en France à l'heure de la mondialisation." In : Sapiro, Gisèle (ed.) 2008. Translatio. Le marché de la traduction en France à l'heure de la mondialisation. Paris : CNRS Editions, pp. 311-332. 
Heilbron, Johan \& Gisèle Sapiro. (2007) "Pour une sociologie de la traduction : bilan et perspectives." In : Heilbron, Johan \& Gisèle Sapiro (Eds.) 2007 Translatio. Le marché de la traduction en France à l'heure de la mondialisation. Paris: CNRS Editions.

HutCHEOn, Linda. (1994) Irony's edge. The theory and politics of irony. London: Routledge.

KRISTEVA, Julia. (1969) Sèméiotikè. Recherches pour une sémanalyse. Paris: Editions du Seuil.

LAROUI, Fouad. (1999) Kijk uit voor parachutisten. Amsterdam : Van Oorschot.

LAROUI, Fouad. (2001a) Judith en Jamal. Amsterdam : Van Oorschot.

LAROUI, Fouad. (2001b) Vreemdeling: aangenaam. Amsterdam : Van Oorschot.

LAROUI, Fouad. (2002a) De tanden van de topograaf. Amsterdam : Van Oorschot.

LAROUI, Fouad. (2002b) Verbannen woorden. Amsterdam : Vassallucci.

LAROUI, Fouad. (2004) De uitvinding van God. Amsterdam : Van Oorschot.

Laroui, Fouad. (2005) Het tragische einde van Philomène Tralala. Amsterdam : Van Oorschot.

LAROUI, Fouad. (2006) Requiem voor de eerste generatie. Breda : De Geus.

LAROuI, Fouad. (2008) De rijkste vrouw van Yorkshire. Breda : De Geus.

LAROUI, Fouad. (2010a) De dag dat Malika niet trouwde : verhalen. Breda De Geus.

LAROUI, Fouad. (2010b) Une année chez les Français. Paris : Julliard.

LAROUI, Fouad. (2012) De kleine bedrieger : een jaar bij de Fransen. Breda De Geus.

LEPPiHALme, Ritva. (1996) "Caught in the frame. A target-culture viewpoint on allusive wordplay." The translator: studies in intercultural communication 2:2, pp. 199-218.

LepPiHAlme, Ritva. (1997) Culture Bumps: An Empirical Approach to the Translation of Allusions. Clevedon: Multilingual Matters Ltd.

PERri, Carmela. (1978) "On alluding." Poetics 7:3, pp. 289-307.

PuCCI, Joseph. (1998) Contemporary versions of allusion The full-knowing reader. Allusion and the power of the reader in the western literary tradition. New Haven \& London : Yale University Press.

RifFATERRE, Michael. (1979) La production du texte. Paris: Seuil.

ROUX-FAUCARD, Geneviève. (2006) "Intertextualité et traduction." Meta 51:1, pp. 98-118.

Schoentjes, Pierre. (2001) Poétique de l'ironie. Paris: Seuil.

VAn CRugten, Alain. (1989) "La récré du traducteur." Meta 34:1, pp. 26-32.

VENUTI, Lawrence. (1995) The translator's invisibility. New York: Routledge.

VENUTI, Lawrence. (1998) The scandals of translation: Towards an ethics of difference. New York: Routledge.

ZabalbeAscoA, Patrick. (2005) "Humor and translation-an interdiscipline." Humor 18:2, pp. 185-207. 


\section{NOTICE BIOGRAPHIQUE / BIONOTE}

KATRIEN LiEVOIS est chargée de cours dans le Département des Traducteurs et Interprètes de la UAntwerpen (Université d'Anvers, Belgique). Elle y enseigne des cours de langue et de civilisation françaises et anime l'axe 'Littérature et/ en Traduction' au sein du groupe de recherche TricS - Translation, interpreting and intercultural Studies. Ses travaux portent sur la pseudo-traduction, la traduction du texte postcolonial, ainsi que sur la traduction de l'ironie et de l'intertextualité dans la littérature française et francophone. Elle est rédacteur en chef de la revue Linguistica Antverpiensia NS - Themes in Translation Studies (https://lans-tts.uantwerpen.be). Site : https://www.uantwerpen.be/nl/ personeel/katrien-lievois/site-personnel/

KATRIEN LIEVOIS is a lecturer of French language and culture at the Department of Applied Linguistics/Translators and Interpreters at the University of Antwerp, where she is also member of the research group Translation, Interpreting and Intercultural Studies (TricS). Her main research interests are (1) the translation of irony and intertextuality, (2) the (Dutch) translations of the African Francophone novel and (3) pseudotranslations. She is also the chief editor of Linguistica Antverpiensia New Series - Themes in Translation Studies (https:// lans-tts.uantwerpen.be). Website: https://www.uantwerpen.be/nl/personeel/ katrien-lievois/site-personnel/ 